Article

\title{
Conformal Maps, Biharmonic Maps, and the Warped Product
}

\section{Seddik Ouakkas * and Djelloul Djebbouri}

Laboratory of Geometry, Analysis, Control and Applications, University de Saida, BP138, En-Nasr, 20000 Saida, Algeria; ddjebbouri20@gmail.com

* Correspondence: seddik.ouakkas@gmail.com; Tel.: +213-663-367-423

Academic Editor: Sadayoshi Kojima

Received: 19 December 2015; Accepted: 23 February 2016; Published: 8 March 2016

\begin{abstract}
In this paper we study some properties of conformal maps between equidimensional manifolds, we construct new example of non-harmonic biharmonic maps and we characterize the biharmonicity of some maps on the warped product manifolds.
\end{abstract}

Keywords: biharmonic map; conformal map; warped product

Mathematics Subject Classifications (2000): 31B30, 58E20, 58E30

\section{Introduction.}

Let $\phi:\left(M^{m}, g\right) \rightarrow\left(N^{n}, h\right)$ be a smooth map between Riemannian manifolds. Then $\phi$ is said to be harmonic if it is a critical point of the energy functional :

$$
E(\phi)=\frac{1}{2} \int_{K}|d \phi|^{2} d v_{g}
$$

for any compact subset $K \subset M$. Equivalently, $\phi$ is harmonic if it satisfies the associated Euler-Lagrange equations :

$$
\tau(\phi)=\operatorname{Tr}_{g} \nabla d \phi=0,
$$

and $\tau(\phi)$ is called the tension field of $\phi$. One can refer to [1-4] for background on harmonic maps. In the context of harmonic maps, the stress-energy tensor was studied in details by Baird and Eells in [5]. The stress-energy tensor for a map $\phi:\left(M^{m}, g\right) \longrightarrow\left(N^{n}, h\right)$ defined by

$$
S(\phi)=e(\phi) g-\phi^{*} h
$$

and the relation between $S(\phi)$ and $\tau(\phi)$ is given by

$$
\operatorname{div} S(\phi)=-h(\tau(\phi), d \phi) .
$$

The map $\phi$ is said to be biharmonic if it is a critical point of the bi-energy functional :

$$
E_{2}(\phi)=\frac{1}{2} \int_{M}|\tau(\phi)|^{2} d v_{g}
$$

Equivalently, $\phi$ is biharmonic if it satisfies the associated Euler-Lagrange equations :

$$
\tau_{2}(\phi)=-\operatorname{Tr}_{g}\left(\nabla^{\phi}\right)^{2} \tau(\phi)-\operatorname{Tr}_{g} R^{N}(\tau(\phi), d \phi) d \phi=0,
$$


where $\nabla^{\phi}$ is the connection in the pull-back bundle $\phi^{-1}(T N)$ and, if $\left(e_{i}\right)_{1 \leq i \leq m}$ is a local orthonormal frame field on $M$, then

$$
\operatorname{Tr}_{g}\left(\nabla^{\phi}\right)^{2} \tau(\phi)=\left(\nabla_{e_{i}}^{\phi} \nabla_{e_{i}}^{\phi}-\nabla_{\nabla_{e_{i}} e_{i}}^{\phi}\right) \tau(\phi),
$$

where we sum over repeated indices. We will call the operator $\tau_{2}(\phi)$, the bi-tension field of the map $\phi$. In analogy with harmonic maps, Jiang In [6] has constructed for a map $\phi$ the stress bi-energy tensor defined by

$$
S_{2}(\phi)=\left(\frac{-1}{2}|\tau(\phi)|^{2}+\operatorname{divh}(\tau(\phi), d \phi)\right) g-2 \operatorname{symh}(\nabla \tau(\phi), d \phi),
$$

where

$$
\operatorname{symh}(\nabla \tau(\phi), d \phi)(X, Y)=\frac{1}{2}\left\{h\left(\nabla_{X} \tau(\phi), d \phi(Y)\right)+h\left(\nabla_{Y} \tau(\phi), d \phi(X)\right)\right\},
$$

for any $X, Y \in \Gamma(T M)$. The stress bi-energy tensor was also studied in [7] and those results could be useful when we study conformal maps. The stress bi-energy tensor of $\phi$ satisfies the following relationship

$$
\operatorname{div}_{2}(\phi)=h\left(\tau_{2}(\phi), d \phi\right) .
$$

Clearly any harmonic map is biharmonic, therefore it is interesting to construct non-harmonic biharmonic maps. In [8] the authors found new examples of biharmonic maps by conformally deforming the domain metric of harmonic ones. While in [9] the author analyzed the behavior of the biharmonic equation under the conformal change the domain metric, she obtained metrics $\widetilde{g}=e^{2 \gamma}$ such that the idendity map $I d:(M, g) \longrightarrow(M, \widetilde{g})$ is biharmonic non-harmonic. Moreover, in [10] the author gave some extensions of the result in [9] together with some further constructions of biharmonic maps. The author in [11] deform conformally the codomain metric in order to render a semi-conformal harmonic map biharmonic. In [12] the authors studied the case where $\phi:\left(M^{n}, g\right) \longrightarrow\left(N^{n}, h\right)$ is a conformal mapping between equidimensional manifolds where they show that a conformal mapping $\phi$ is biharmonic if and only if the gradient of its dilation satisfies a second order elliptic partial differential equation. We can refer the reader to [13], for a survey of biharmonic maps. In the first section of this paper, we present some properties for a conformal mapping $\phi:\left(M^{n}, g\right) \longrightarrow\left(N^{n}, h\right)$, we prove that the stress bi-energy tensor depend only on the dilation (Theorem 1) and we calculate the bitension field of $\phi$ (Theorem 2). In the last section we study the biharmonicity of some maps on the warped product (Theorem 4 and 5), with this setting we obtain new examples of biharmonic non-harmonic maps.

\section{Some properties for conformal maps.}

We study conformal maps between equidimensional manifolds of the same dimension $n \geq 3$. Note that by a result in [12], any such map can have no critical points and so is a local conformal diffeomorphism. Recall that a mapping $\phi:\left(M^{n}, g\right) \rightarrow\left(N^{n}, h\right)$ is called conformal if there exist a $C^{\infty}$ function $\lambda: M \rightarrow \mathbb{R}_{+}^{*}$ such that for any $X, Y \in \Gamma(T M)$ :

$$
h(d \phi(X), d \phi(Y))=\lambda^{2} g(X, Y) .
$$

The function $\lambda$ is called the dilation for the map $\phi$. The tension field and the stress energy tensor for a conformal map are given by (see [14]):

Proposition 1. Let $\phi:\left(M^{n}, g\right) \rightarrow\left(N^{n}, h\right)$ be a conformal map of dilation $\lambda$, we have

$$
\text { (i) } \operatorname{div} S(\phi)=(n-2) \lambda^{2} d \ln \lambda \text {, }
$$

(ii) $\operatorname{divh}(\tau(\phi), d \phi)=(2-n)\left(2 \lambda^{2}|\operatorname{grad} \ln \lambda|^{2}+\lambda^{2} \Delta \ln \lambda\right)$.

(iii) $\tau(\phi)=(2-n) d \phi($ grad $\ln \lambda)$.

(iv) $|\tau(\phi)|^{2}=(2-n)^{2} \lambda^{2}|\operatorname{grad} \ln \lambda|^{2}$. 
Note that the conformal map $\phi:\left(M^{n}, g\right) \rightarrow\left(N^{n}, h\right)$ of dilation $\lambda$ is harmonic if and only if $n=2$ or the dilation $\lambda$ is constant.

In the first, wa calculate the stress bi-energy tensor for a conformal map $\phi$ when we prove that $S_{2}(\phi)$ depend only the dilation.

Theorem 1. Let $\phi:\left(M^{n}, g\right) \rightarrow\left(N^{n}, h\right)$ be a conformal map with dilation $\lambda$, then we have

$$
S_{2}(\phi)=(2-n) \lambda^{2}\left\{\left(\frac{n-2}{2}|\operatorname{grad} \ln \lambda|^{2}+\Delta \ln \lambda\right) g-2 \nabla d \ln \lambda\right\},
$$

and the trace of $S_{2}(\phi)$ is given by

$$
\operatorname{Tr} S_{2}(\phi)=-(2-n)^{2} \lambda^{2}\left\{\frac{n}{2}|\operatorname{grad} \ln \lambda|^{2}+\Delta \ln \lambda\right\}
$$

To prove Theorem 1, we need the following Lemma :

Lemma 1. Let $\phi:\left(M^{n}, g\right) \rightarrow\left(N^{n}, h\right)$ be a conformal map with dilation $\lambda$, then for any function $f \in C^{\infty}(M)$ and for any $X, Y \in \Gamma(T M)$, we have

$$
\begin{aligned}
h\left(\nabla_{X} d \phi(\operatorname{grad} f), d \phi(Y)\right) & =\lambda^{2}(X(\ln \lambda) Y(f)-Y(\ln \lambda) X(f)) \\
& +\lambda^{2} \nabla d f(X, Y)+\lambda^{2} d \ln \lambda(\operatorname{grad} f) g(X, Y) .
\end{aligned}
$$

Proof of Lemma 1. Let $f \in C^{\infty}(M)$, for any $X, Y \in \Gamma(T M)$, we have

$$
\begin{aligned}
h\left(\nabla_{X} d \phi(\operatorname{grad} f), d \phi(Y)\right) & =X\left(\lambda^{2} g(\operatorname{grad} f, Y)\right)-h\left(d \phi(\operatorname{grad} f), \nabla_{X} d \phi(Y)\right) \\
& =X\left(\lambda^{2}\right) g(\operatorname{grad} f, Y)+\lambda^{2} g\left(\nabla_{X} \operatorname{grad} f, Y\right)+\lambda^{2} g\left(\operatorname{grad} f, \nabla_{X} Y\right) \\
& -h(d \phi(\operatorname{grad} f), \nabla d \phi(X, Y))-h\left(d \phi(\operatorname{grad} f), d \phi\left(\nabla_{X} Y\right)\right) \\
& =X\left(\lambda^{2}\right) \operatorname{g}(\operatorname{grad} f, Y)+\lambda^{2} g\left(\nabla_{X} \operatorname{grad} f, Y\right)+\lambda^{2} g\left(\operatorname{grad} f, \nabla_{X} Y\right) \\
& -h(d \phi(\operatorname{grad} f), \nabla d \phi(X, Y))-\lambda^{2} g\left(\operatorname{grad} f, \nabla_{X} Y\right) .
\end{aligned}
$$

Note that

$$
g\left(\nabla_{X} \operatorname{grad} f, Y\right)=\nabla d f(X, Y)
$$

then we obtain

$$
h\left(\nabla_{X} d \phi(\operatorname{gradf}), d \phi(Y)\right)=2 \lambda^{2} X(\ln \lambda) Y(f)+\lambda^{2} \nabla d f(X, Y)-h(d \phi(\operatorname{grad} f), \nabla d \phi(X, Y)) .
$$

By similary, we have

$$
h\left(\nabla_{Y} d \phi(\operatorname{grad} f), d \phi(X)\right)=2 \lambda^{2} Y(\ln \lambda) X(f)+\lambda^{2} \nabla d f(X, Y)-h(d \phi(\operatorname{grad} f), \nabla d \phi(X, Y)) .
$$

Then, we deduce that

$$
\begin{aligned}
h\left(\nabla_{X} d \phi(\operatorname{grad} f), d \phi(Y)\right) & =h\left(d \phi(X), \nabla_{Y} d \phi(\operatorname{grad} f)\right) \\
& +2 \lambda^{2}(X(\ln \lambda) Y(f)-Y(\ln \lambda) X(f)) .
\end{aligned}
$$


For the term $h\left(d \phi(X), \nabla_{Y} d \phi(\operatorname{grad} f)\right)$, we have

$$
\begin{aligned}
h\left(\nabla_{Y} d \phi(\operatorname{grad} f), d \phi(X)\right) & =h(\nabla d \phi(\operatorname{grad} f, Y), d \phi(X))+\lambda^{2} g\left(\nabla_{Y} \operatorname{grad} f, X\right) \\
& =h\left(\nabla_{\text {gradf }} d \phi(Y), d \phi(X)\right)-\lambda^{2} g\left(\nabla_{\text {gradf }} Y, X\right) \\
& +\lambda^{2} g\left(\nabla_{Y} \operatorname{grad} f, X\right) \\
& =\operatorname{grad} f\left(\lambda^{2} g(X, Y)\right)-h\left(\nabla_{\operatorname{gradf}} d \phi(X), d \phi(Y)\right) \\
& -\lambda^{2} g\left(\nabla_{\operatorname{grad}} Y, X\right)+\lambda^{2} g\left(\nabla_{Y} \operatorname{grad} f, X\right) \\
& =2 \lambda^{2} d \ln \lambda(\operatorname{grad} f) g(X, Y)-h(\nabla d \phi(X, \operatorname{grad} f), d \phi(Y)) \\
& +\lambda^{2} g\left(\nabla_{Y} \operatorname{grad} f, X\right) .
\end{aligned}
$$

We deduce that

$$
\begin{aligned}
h\left(\nabla_{Y} d \phi(\operatorname{grad} f), d \phi(X)\right) & =-h\left(\nabla_{X} d \phi(\operatorname{grad} f), d \phi(Y)\right)+2 \lambda^{2} \nabla d f(X, Y) \\
& +2 \lambda^{2} d \ln \lambda(\operatorname{grad} f) g(X, Y) .
\end{aligned}
$$

Finally, if we replace (13) in (12), we obtain

$$
\begin{aligned}
h\left(\nabla_{X} d \phi(\operatorname{grad} f), d \phi(Y)\right) & =\lambda^{2}(X(\ln \lambda) Y(f)-Y(\ln \lambda) X(f)) \\
& +\lambda^{2} \nabla d f(X, Y)+\lambda^{2} d \ln \lambda(\operatorname{grad} f) g(X, Y) .
\end{aligned}
$$

This completes the proof of Lemma 1.

Remark 1. Let $\phi:\left(M^{n}, g\right) \rightarrow\left(N^{n}, h\right)$ be a conformal map with dilation $\lambda$, then if we consider $f=\ln \lambda$, the equation (11) gives

$$
h\left(\nabla_{X} d \phi(\operatorname{grad} \ln \lambda), d \phi(Y)\right)=\lambda^{2}\left(\nabla d \ln \lambda(X, Y)+|\operatorname{grad} \ln \lambda|^{2} g(X, Y)\right) .
$$

Proof of Theorem 1. By definition, the stress bi-energy tensor is given by :

$$
S_{2}(\phi)=\left(-\frac{1}{2}|\tau(\phi)|^{2}+\operatorname{divh}(\tau(\phi), d \phi)\right) g-2 \operatorname{symh}(\nabla \tau(\phi), d \phi) .
$$

Using the equations (2) et (4) for the Proposition 1, we have

$$
-\frac{1}{2}|\tau(\phi)|^{2}+\operatorname{divh}(\tau(\phi), d \phi)=(2-n) \lambda^{2}\left(\frac{n+2}{2}|\operatorname{grad} \ln \lambda|^{2}+\Delta \ln \lambda\right) .
$$

Calculate now $\operatorname{symh}(\nabla \tau(\phi), d \phi)$, we have by definition for any $X, Y \in \Gamma(T M)$

$$
\begin{aligned}
\operatorname{symh}(\nabla \tau(\phi), d \phi)(X, Y) & =\frac{1}{2}\left(h\left(\nabla_{X} \tau(\phi), d \phi(Y)\right)+h\left(\nabla_{Y} \tau(\phi), d \phi(X)\right)\right) \\
& =\frac{2-n}{2}\left(h\left(\nabla_{X} d \phi(\operatorname{grad} \ln \lambda), d \phi(Y)\right)+h\left(\nabla_{Y}(\operatorname{grad} \ln \lambda), d \phi(X)\right)\right) .
\end{aligned}
$$

By Lemma 1, we have

$$
h\left(\nabla_{X} d \phi(\operatorname{grad} \ln \lambda), d \phi(Y)\right)=\lambda^{2}\left(\nabla d \ln \lambda(X, Y)+|\operatorname{grad} \ln \lambda|^{2} g(X, Y)\right)
$$

and

$$
h\left(\nabla_{Y} d \phi(\operatorname{grad} \ln \lambda), d \phi(X)\right)=\lambda^{2}\left(\nabla d \ln \lambda(X, Y)+|\operatorname{grad} \ln \lambda|^{2} g(X, Y)\right)
$$


then

$$
\operatorname{symh}(\nabla \tau(\phi), d \phi)(X, Y)=(2-n) \lambda^{2}\left(\nabla d \ln \lambda(X, Y)+|\operatorname{grad} \ln \lambda|^{2} g(X, Y)\right) .
$$

If we substitute (15) and (16) in (14), we conclude that

$$
S_{2}(\phi)=(2-n) \lambda^{2}\left\{\left(\frac{n-2}{2}|g r a d \ln \lambda|^{2}+\Delta \ln \lambda\right) g-2 \nabla d \ln \lambda\right\}
$$

Calculate now the trace of stress bi-energy tensor. Let $\left(e_{i}\right)_{1 \leq i \leq n}$ be an orthonormal frame on $M$, we have

$$
\begin{aligned}
\operatorname{Tr}_{g} S_{2}(\phi) & =S_{2}(\phi)\left(e_{i}, e_{i}\right) \\
& =(2-n) \lambda^{2}\left(\frac{n-2}{2}|g r a d \ln \lambda|^{2}+\Delta \ln \lambda\right) g\left(e_{i}, e_{i}\right) \\
& -2(2-n) \lambda^{2} \nabla d \ln \lambda\left(e_{i}, e_{i}\right) \\
& =(2-n) n \lambda^{2}\left(\frac{n-2}{2}|g r a d \ln \lambda|^{2}+\Delta \ln \lambda\right) \\
& -2(2-n) \lambda^{2}(\Delta \ln \lambda) \\
& =(2-n) \lambda^{2}\left\{\frac{n(n-2)}{2}|g r a d \ln \lambda|^{2}+(n-2) \Delta \ln \lambda\right\} .
\end{aligned}
$$

Then

$$
\operatorname{Tr}_{2}(\phi)=-(2-n)^{2} \lambda^{2}\left\{\frac{n}{2}|\operatorname{grad} \ln \lambda|^{2}+\Delta \ln \lambda\right\}
$$

By calculating the Laplacian of the function $\lambda^{\frac{n}{2}}$ and by using

$$
\Delta \lambda^{\frac{n}{2}}=\frac{n}{2} \lambda^{\frac{n}{2}}\left(\frac{n}{2}|\operatorname{grad} \ln \lambda|^{2}+\Delta \ln \lambda\right)
$$

we obtain immediately the following corollary

Corollary 1. Let $\phi:\left(M^{n}, g\right) \rightarrow\left(N^{n}, h\right),(n \neq 2)$ to be a conformal map of dilation $\lambda$, then the trace of $S_{2}(\phi)$ is zero if and only if the function $\lambda^{\frac{n}{2}}$ is harmonic.

The bi-tension field of the conformal map is given by

Theorem 2. Let $\phi:\left(M^{n}, g\right) \rightarrow\left(N^{n}, h\right),(n \geq 3)$ to be a conformal map of dilation $\lambda$, then bi-tension field of $\phi$ is given by:

$$
\tau_{2}(\phi)=(n-2) d \phi(H)
$$

where

$$
\begin{aligned}
H & =\operatorname{grad} \Delta \ln \lambda-\frac{(n-6)}{2} \operatorname{grad}\left(|\operatorname{grad} \ln \lambda|^{2}\right)+2 \operatorname{Ricci}^{M}(\operatorname{grad} \ln \lambda) \\
& -\left(2(\Delta \ln \lambda)+(n-2)|\operatorname{grad} \ln \lambda|^{2}\right) \operatorname{grad} \ln \lambda .
\end{aligned}
$$

Remark 2. A. Balmus in [9] studied the case where $\phi=I d_{M}$, she obtained the biharmonicity of the identity map from $(M, g)$ onto $\left(M, \lambda^{2} g\right)$, this case was also studied in [15].

To prove the Theorem 2, we need two Lemmas. In the first Lemma, we give a simple formula of the term $\operatorname{Tr}_{g}\left(\nabla^{\phi}\right)^{2} d \phi(\operatorname{grad} \gamma)$ for a conformal map $\phi:\left(M^{n}, g\right) \rightarrow\left(N^{n}, h\right)(n \geq 3)$ of dilation $\lambda$ and for any function $\gamma \in C^{\infty}(M)$.

Lemma 2. Let $\phi:\left(M^{n}, g\right) \rightarrow\left(N^{n}, h\right)(n \geq 3)$ to be a conformal map of dilation $\lambda$, then for any function $\gamma \in C^{\infty}(M)$, we have

$$
\begin{aligned}
\operatorname{Tr}_{g}\left(\nabla^{\phi}\right)^{2} d \phi(\operatorname{grad} \gamma) & =d \phi(\operatorname{grad} \Delta \gamma)+4 d \phi\left(\nabla_{\operatorname{grad}} \ln \operatorname{grad} \gamma\right)+d \phi\left(\operatorname{Ricci}^{M}(\operatorname{grad} \gamma)\right) \\
& +(\Delta \ln \lambda) d \phi(\operatorname{grad} \gamma)-2(\Delta \gamma) d \phi(\operatorname{grad} \ln \lambda) \\
& -(n-2) d \ln \lambda(\operatorname{grad} \gamma) d \phi(\operatorname{grad} \ln \lambda) .
\end{aligned}
$$


Proof of Lemma 2. Let $\gamma \in C^{\infty}(M)$, by definition, we have

$$
\operatorname{Tr}_{g}\left(\nabla^{\phi}\right)^{2} d \phi(\operatorname{grad} \gamma)=\nabla_{e_{i}}^{\phi} \nabla_{e_{i}}^{\phi} d \phi(\operatorname{grad} \gamma)-\nabla_{\nabla_{e_{i}} e_{i}}^{\phi} d \phi(\operatorname{grad} \gamma)
$$

(Here henceforth we sum over repeated indices.) Let us start with the calculation of the term $\nabla_{e_{i}}^{\phi} \nabla_{e_{i}}^{\phi} d \phi(g r a d \gamma)$, we have

$$
\nabla_{e_{i}}^{\phi} d \phi(\operatorname{grad} \gamma)=\nabla d \phi\left(e_{i}, \operatorname{grad} \gamma\right)+d \phi\left(\nabla_{e_{i}} \operatorname{grad} \gamma\right)
$$

It is known that (see [16])

$$
\nabla d \phi\left(e_{i}, \operatorname{grad} \gamma\right)=e_{i}(\ln \lambda) d \phi(\operatorname{grad} \gamma)+d \ln \lambda(\operatorname{grad} \gamma) d \phi\left(e_{i}\right)-e_{i}(\gamma) d \phi(\operatorname{grad} \ln \lambda)
$$

then

$$
\begin{aligned}
\nabla_{e_{i}}^{\phi} d \phi(\operatorname{grad} \gamma) & =e_{i}(\ln \lambda) d \phi(\operatorname{grad} \gamma)+d \ln \lambda(\operatorname{grad} \gamma) d \phi\left(e_{i}\right) \\
& -e_{i}(\gamma) d \phi(\operatorname{grad} \ln \lambda)+d \phi\left(\nabla_{e_{i}} \operatorname{grad} \gamma\right) .
\end{aligned}
$$

It follows that

$$
\begin{aligned}
\nabla_{e_{i}}^{\phi} \nabla_{e_{i}}^{\phi} d \phi(\operatorname{grad} \gamma) & =\nabla_{e_{i}}^{\phi}\left\{e_{i}(\ln \lambda) d \phi(\operatorname{grad} \gamma)\right\}+\nabla_{e_{i}}^{\phi}\left\{d \ln \lambda(\operatorname{grad} \gamma) d \phi\left(e_{i}\right)\right\} \\
& -\nabla_{e_{i}}^{\phi}\left\{e_{i}(\gamma) d \phi(\operatorname{grad} \ln \lambda)\right\}+\nabla_{e_{i}}^{\phi} d \phi\left(\nabla_{e_{i}} \operatorname{grad} \gamma\right) .
\end{aligned}
$$

We will study term by term the right-hand of this expression. For the first term $\nabla_{e_{i}}^{\phi}\left\{e_{i}(\ln \lambda) d \phi(\operatorname{grad} \gamma)\right\}$, we have

$$
\nabla_{e_{i}}^{\phi}\left\{e_{i}(\ln \lambda) d \phi(\operatorname{grad} \gamma)\right\}=e_{i}(\ln \lambda) \nabla_{e_{i}}^{\phi} d \phi(\operatorname{grad} \gamma)+e_{i}\left(e_{i}(\ln \lambda)\right) d \phi(\operatorname{grad} \gamma) .
$$

By using the equation (20), we deduce that

$$
\begin{aligned}
\nabla_{e_{i}}^{\phi}\left\{e_{i}(\ln \lambda) d \phi(\operatorname{grad} \gamma)\right\} & =e_{i}(\ln \lambda) e_{i}(\ln \lambda) d \phi(\operatorname{grad} \gamma)+e_{i}(\ln \lambda) d \ln \lambda(\operatorname{grad} \gamma) d \phi\left(e_{i}\right) \\
& -e_{i}(\ln \lambda) e_{i}(\gamma) d \phi(\operatorname{grad} \ln \lambda)+e_{i}(\ln \lambda) d \phi\left(\nabla_{e_{i}} \operatorname{grad} \gamma\right) \\
& +e_{i}\left(e_{i}(\ln \lambda)\right) d \phi(\operatorname{grad} \gamma),
\end{aligned}
$$

then, we obtain

$$
\begin{aligned}
\nabla_{e_{i}}^{\phi}\left\{e_{i}(\ln \lambda) d \phi(\operatorname{grad} \gamma)\right\} & =|\operatorname{grad} \ln \lambda|^{2} d \phi(\operatorname{grad} \gamma)+d \phi\left(\nabla_{\operatorname{grad} \ln \lambda} \operatorname{grad} \gamma\right) \\
& +e_{i}\left(e_{i}(\ln \lambda)\right) d \phi(\operatorname{grad} \gamma)
\end{aligned}
$$

For the second term $\nabla_{e_{i}}^{\phi}\left\{d \ln \lambda(\operatorname{grad} \gamma) d \phi\left(e_{i}\right)\right\}$, a similar calculation gives

$$
\begin{aligned}
\nabla_{e_{i}}^{\phi}\left\{d \ln \lambda(\operatorname{grad} \gamma) d \phi\left(e_{i}\right)\right\} & =d \ln \lambda(\operatorname{grad} \gamma) \nabla_{e_{i}}^{\phi} d \phi\left(e_{i}\right)+e_{i}\{g(\operatorname{grad} \ln \lambda, \operatorname{grad} \gamma)\} d \phi\left(e_{i}\right) \\
& =d \ln \lambda(\operatorname{grad} \gamma) \nabla_{e_{i}}^{\phi} d \phi\left(e_{i}\right)+g\left(\nabla_{e_{i}} \operatorname{grad} \ln \lambda, \operatorname{grad} \gamma\right) d \phi\left(e_{i}\right) \\
& +g\left(\operatorname{grad} \ln \lambda, \nabla_{e_{i}} \operatorname{grad} \gamma\right) d \phi\left(e_{i}\right) \\
& =d \ln \lambda(\operatorname{grad} \gamma) \nabla_{e_{i}}^{\phi} d \phi\left(e_{i}\right)+g\left(\nabla_{\operatorname{grad} \gamma} \operatorname{grad} \ln \lambda, e_{i}\right) d \phi\left(e_{i}\right) \\
& +g\left(\nabla_{\operatorname{grad} \ln \lambda} \operatorname{grad} \gamma, e_{i}\right) d \phi\left(e_{i}\right),
\end{aligned}
$$


it follows that

$$
\begin{aligned}
\nabla_{e_{i}}^{\phi}\left\{d \ln \lambda(\operatorname{grad} \gamma) d \phi\left(e_{i}\right)\right\} & =d \ln \lambda(\operatorname{grad} \gamma) \nabla_{e_{i}}^{\phi} d \phi\left(e_{i}\right)+d \phi\left(\nabla_{\operatorname{grad} \gamma} \operatorname{grad} \ln \lambda\right) \\
& +d \phi\left(\nabla_{\operatorname{grad} \ln \lambda} \operatorname{grad} \gamma\right) .
\end{aligned}
$$

For the third term $\nabla_{e_{i}}^{\phi}\left\{e_{i}(\gamma) d \phi(\operatorname{grad} \ln \lambda)\right\}$, by using the same calculation method and the equation (20), we have

$$
\begin{aligned}
\nabla_{e_{i}}^{\phi}\left\{e_{i}(\gamma) d \phi(\operatorname{grad} \ln \lambda)\right\} & =e_{i}(\gamma) \nabla_{e_{i}}^{\phi} d \phi(\operatorname{grad} \ln \lambda)+e_{i}\left(e_{i}(\gamma)\right) d \phi(\operatorname{grad} \ln \lambda) \\
& =e_{i}(\gamma) e_{i}(\ln \lambda) d \phi(\operatorname{grad} \ln \lambda)+e_{i}(\gamma) d \ln \lambda(\operatorname{grad} \ln \lambda) d \phi\left(e_{i}\right) \\
& -e_{i}(\gamma) e_{i}(\ln \lambda) d \phi(\operatorname{grad} \ln \lambda)+e_{i}(\gamma) d \phi\left(\nabla_{e_{i}} \operatorname{grad} \ln \lambda\right) \\
& +e_{i}\left(e_{i}(\gamma)\right) d \phi(\operatorname{grad} \ln \lambda)
\end{aligned}
$$

which gives us

$$
\begin{aligned}
\nabla_{e_{i}}^{\phi}\left\{e_{i}(\gamma) d \phi(\operatorname{grad} \ln \lambda)\right\} & =|\operatorname{grad} \ln \lambda|^{2} d \phi(\operatorname{grad} \gamma)+d \phi\left(\nabla_{\operatorname{grad} \gamma} \operatorname{grad} \ln \lambda\right) \\
& +e_{i}\left(e_{i}(\gamma)\right) d \phi(\operatorname{grad} \ln \lambda) .
\end{aligned}
$$

Now let us look at the last term $\nabla_{e_{i}}^{\phi} d \phi\left(\nabla_{e_{i}}\right.$ grad $\left.\gamma\right)$, a simple calculation gives

$$
\begin{aligned}
\nabla_{e_{i}}^{\phi} d \phi\left(\nabla_{e_{i}} \operatorname{grad} \gamma\right) & =e_{i}(\ln \lambda) d \phi\left(\nabla_{e_{i}} \operatorname{grad} \gamma\right)+d \ln \lambda\left(\nabla_{e_{i}} \operatorname{grad} \gamma\right) d \phi\left(e_{i}\right) \\
& -g\left(e_{i}, \nabla_{e_{i}} \operatorname{grad} \gamma\right) d \phi(\operatorname{grad} \ln \lambda)+d \phi\left(\nabla_{e_{i}} \nabla_{e_{i}} g r a d \gamma\right) \\
& =2 d \phi\left(\nabla_{\operatorname{grad} \ln \lambda} \operatorname{grad} \gamma\right)-(\Delta \gamma) d \phi(\operatorname{grad} \ln \lambda) \\
& +d \phi\left(\nabla_{e_{i}} \nabla_{e_{i}} \operatorname{grad} \gamma\right)
\end{aligned}
$$

then

$$
\begin{aligned}
\nabla_{e_{i}}^{\phi} d \phi\left(\nabla_{e_{i}} \operatorname{grad} \gamma\right. & =d \phi\left(\nabla_{e_{i}} \nabla_{e_{i}} \operatorname{grad} \gamma\right)+2 d \phi\left(\nabla_{\operatorname{grad} \ln \lambda} \operatorname{grad} \gamma\right) \\
& -(\Delta \gamma) d \phi(\operatorname{grad} \ln \lambda) .
\end{aligned}
$$

If we replace (22), (23), (24) and (25) in (21), we obtain

$$
\begin{aligned}
\nabla_{e_{i}}^{\phi} \nabla_{e_{i}}^{\phi} d \phi(\operatorname{grad} \gamma) & =4 d \phi\left(\nabla_{\operatorname{grad} \ln \lambda} \operatorname{grad} \gamma\right)+e_{i}\left(e_{i}(\ln \lambda)\right) d \phi(\operatorname{grad} \gamma) \\
& +d \ln \lambda(\operatorname{grad} \gamma) \nabla_{e_{i}}^{\phi} d \phi\left(e_{i}\right)-e_{i}\left(e_{i}(\gamma)\right) d \phi(\operatorname{grad} \ln \lambda) \\
& +d \phi\left(\nabla_{e_{i}} \nabla_{e_{i}} \operatorname{grad} \gamma\right)-(\Delta \gamma) d \phi(\operatorname{grad} \ln \lambda) .
\end{aligned}
$$

To complete the proof, it remains to investigate the term $\nabla_{\nabla_{e_{i}} e_{i}}^{\phi} d \phi(\operatorname{grad} \gamma)$, we have

$$
\nabla_{\nabla_{e_{i}} e_{i}}^{\phi} d \phi(\operatorname{grad} \gamma)=\nabla d \phi\left(\nabla_{e_{i}} e_{i}, \operatorname{grad} \gamma\right)+d \phi\left(\nabla_{\nabla_{e_{i}} e_{i}} \operatorname{grad} \gamma\right)
$$

Therefore, by using the equation (20), we obtain

$$
\begin{aligned}
\nabla_{\nabla_{e_{i}} e_{i}}^{\phi} d \phi(\operatorname{grad} \gamma) & =\nabla_{e_{i}} e_{i}(\ln \lambda) d \phi(\operatorname{grad} \gamma)+d \ln \lambda(\operatorname{grad} \gamma) d \phi\left(\nabla_{e_{i}} e_{i}\right) \\
& -\nabla_{e_{i}} e_{i}(\gamma) d \phi(\operatorname{grad} \ln \lambda)+d \phi\left(\nabla_{\nabla_{e_{i}} e_{i} \operatorname{grad} \gamma}\right) .
\end{aligned}
$$


By substituting (26) and (27) in (19), we deduce

$$
\begin{aligned}
\operatorname{Trg}_{g}\left(\nabla^{\phi}\right)^{2} d \phi(\operatorname{grad} \gamma) & =\nabla_{e_{i}}^{\phi} \nabla_{e_{i}}^{\phi} d \phi(\operatorname{grad} \gamma)-\nabla_{\nabla_{e_{i}} e_{i}}^{\phi} d \phi(\operatorname{grad} \gamma) \\
& =d \phi\left(\operatorname{Tr}_{g} \nabla^{2} \operatorname{grad} \gamma\right)+4 d \phi\left(\nabla_{\operatorname{grad} \ln \lambda} \operatorname{grad} \gamma\right) \\
& +(\Delta \ln \lambda) d \phi(\operatorname{grad} \gamma)+d \ln \lambda(\operatorname{grad} \gamma) \tau(\phi) \\
& -2(\Delta \gamma) d \phi(\operatorname{grad} \ln \lambda) .
\end{aligned}
$$

Finally, using the fact that (see [11])

$$
\operatorname{Tr}_{g} \nabla^{2} \operatorname{grad} \gamma=\operatorname{grad} \Delta \gamma+\operatorname{Ricci}^{M}(\operatorname{grad} \gamma)
$$

and

$$
\tau(\phi)=(2-n) d \phi(\operatorname{grad} \ln \lambda),
$$

we conclude that

$$
\begin{aligned}
\operatorname{Tr}_{g}\left(\nabla^{\phi}\right)^{2} d \phi(\operatorname{grad} \gamma) & =d \phi(\operatorname{grad} \Delta \gamma)+4 d \phi\left(\nabla_{\operatorname{grad} \ln \lambda} \operatorname{grad} \gamma\right)+d \phi\left(\operatorname{Ricci}^{M}(\operatorname{grad} \gamma)\right) \\
& +(\Delta \ln \lambda) d \phi(\operatorname{grad} \gamma)-2(\Delta \gamma) d \phi(\operatorname{grad} \ln \lambda) \\
& -(n-2) d \ln \lambda(\operatorname{grad} \gamma) d \phi(\operatorname{grad} \ln \lambda) .
\end{aligned}
$$

This completes the proof of Lemma 2. Now, in the second Lemma, we will calculate $\operatorname{Tr}_{g} R^{N}(d \phi(\operatorname{grad} \gamma), d \phi) d \phi$ for a conformal maps $\phi:\left(M^{n}, g\right) \rightarrow\left(N^{n}, h\right)(n \geq 3)$ of dilation $\lambda$ and for any function $\gamma \in C^{\infty}(M)$

Lemma 3. Let $\phi:\left(M^{n}, g\right) \rightarrow\left(N^{n}, h\right)(n \geq 3)$ to be a conformal map of dilation $\lambda$, then for any function $\gamma \in C^{\infty}(M)$, we have

$$
\begin{aligned}
\operatorname{Tr}_{g} R^{N}(d \phi(\operatorname{grad} \gamma), d \phi) d \phi & =d \phi\left(\operatorname{Ricci}^{M}(\operatorname{grad} \gamma)\right)-(n-2) d \phi\left(\nabla_{\operatorname{grad} \gamma} \operatorname{grad} \ln \lambda\right) \\
& -\left(\Delta \ln \lambda+(n-2)|\operatorname{grad} \ln \lambda|^{2}\right) d \phi(\operatorname{grad} \gamma) \\
& +(n-2) d \ln \lambda(\operatorname{grad} \gamma) d \phi(\operatorname{grad} \ln \lambda)
\end{aligned}
$$

Proof of Lemma 3. Let $\gamma \in C^{\infty}(M)$, by definition we have

$$
\operatorname{Tr}_{g} R^{N}(d \phi(\operatorname{grad} \gamma), d \phi) d \phi=R^{N}\left(d \phi(\operatorname{grad} \gamma), d \phi\left(e_{i}\right)\right) d \phi\left(e_{i}\right)
$$

but we know that (see [16])

$$
\begin{aligned}
\operatorname{Ric}^{N}(d \phi(X), d \phi(Y)) & =\operatorname{Ric}^{M}(X, Y)+(n-2) X(\ln \lambda) Y(\ln \lambda) \\
& -(n-2)|\operatorname{grad} \ln \lambda|^{2} g(X, Y) \\
& -(n-2) \nabla d \ln \lambda(X, Y)-(\Delta \ln \lambda) g(X, Y) .
\end{aligned}
$$

Then

$$
\begin{aligned}
\operatorname{Ric}^{N}\left(d \phi(\operatorname{grad} \gamma), d \phi\left(e_{i}\right)\right) & =\operatorname{Ric}^{M}\left(\operatorname{grad} \gamma, e_{i}\right)+(n-2) \operatorname{grad} \gamma(\ln \lambda) e_{i}(\ln \lambda) \\
& -(n-2)|\operatorname{grad} \ln \lambda|^{2} \operatorname{g}\left(\operatorname{grad} \gamma, e_{i}\right) \\
& \left.-(n-2) \nabla d \ln \lambda\left(\operatorname{grad} \gamma, e_{i}\right)-(\Delta \ln \lambda) \operatorname{grad} \gamma, e_{i}\right)
\end{aligned}
$$


it follows that

$$
\begin{aligned}
\operatorname{Ric}^{N}\left(d \phi(\operatorname{grad} \gamma), d \phi\left(e_{i}\right)\right) & =\operatorname{Ric}^{M}\left(\operatorname{grad} \gamma, e_{i}\right)+(m-2) d \ln \lambda(\operatorname{grad} \gamma) e_{i}(\ln \lambda) \\
& -(n-2)|\operatorname{grad} \ln \lambda|^{2} e_{i}(\gamma)-(n-2) \nabla d \ln \lambda\left(\operatorname{grad} \gamma, e_{i}\right) \\
& -(\Delta \ln \lambda) e_{i}(\gamma) .
\end{aligned}
$$

If we replace (30) in (29), we deduce that

$$
\begin{aligned}
\operatorname{Tr}_{g} R^{N}(d \phi(\operatorname{grad} \gamma), d \phi) d \phi & =R^{N}\left(d \phi(\operatorname{grad} \gamma), d \phi\left(e_{i}\right)\right) d \phi\left(e_{i}\right) \\
& =d \phi\left(\operatorname{Ricci}^{M}(\operatorname{grad} \gamma)\right)+(n-2) d \ln \lambda(\operatorname{grad} \gamma) d \phi(\operatorname{grad} \ln \lambda) \\
& -(n-2)|\operatorname{grad} \ln \lambda|^{2} d \phi(\operatorname{grad} \gamma)-(n-2) \nabla d \ln \lambda\left(\operatorname{grad} \gamma, e_{i}\right) d \phi\left(e_{i}\right) \\
& -(\Delta \ln \lambda) d \phi(\operatorname{grad} \gamma) .
\end{aligned}
$$

To complete the proof, we will simplify the term $\nabla d \ln \lambda\left(\operatorname{grad} \gamma, e_{i}\right) d \phi\left(e_{i}\right)$, we obtain

$$
\begin{aligned}
\nabla d \ln \lambda\left(\operatorname{grad} \gamma, e_{i}\right) d \phi\left(e_{i}\right) & \left.=\left\{e_{i}(\operatorname{grad} \ln \lambda, \operatorname{grad} \gamma)\right)-d \ln \lambda\left(\nabla_{e_{i}} \operatorname{grad} \gamma\right)\right\} d \phi\left(e_{i}\right) \\
& =g\left(\nabla_{e_{i}} \operatorname{grad} \ln \lambda, \operatorname{grad} \gamma\right) d \phi\left(e_{i}\right) \\
& =g\left(\nabla_{\operatorname{grad} \gamma} \operatorname{grad} \ln \lambda, e_{i}\right) d \phi\left(e_{i}\right) \\
& =d \phi\left(\nabla_{\operatorname{grad} \gamma} \operatorname{grad} \ln \lambda\right),
\end{aligned}
$$

which finally gives

$$
\begin{aligned}
\operatorname{Tr}_{g} R^{N}(d \phi(\operatorname{grad} \gamma), d \phi) d \phi & =d \phi\left(\operatorname{Ricci}^{M}(\operatorname{grad} \gamma)\right)-(n-2) d \phi\left(\nabla_{\operatorname{grad} \gamma} \operatorname{grad} \ln \lambda\right) \\
& -\left(\Delta \ln \lambda+(n-2)|\operatorname{grad} \ln \lambda|^{2}\right) d \phi(\operatorname{grad} \gamma) \\
& +(n-2) d \ln \lambda(\operatorname{grad} \gamma) d \phi(\operatorname{grad} \ln \lambda) .
\end{aligned}
$$

This completes the proof of Lemma 3. We are now able to prove Theorem 2.

Proof of Theorem 2. By definition, the bi-tension field is given by

$$
\tau_{2}(\phi)=-\operatorname{Tr}_{g}\left(\nabla^{\phi}\right)^{2} \tau(\phi)-\operatorname{Tr}_{g} R^{N}(\tau(\phi), d \phi) d \phi .
$$

The tension field of the conformal map $\phi$ is given by

$$
\tau(\phi)=(2-n) d \phi(\operatorname{grad} \ln \lambda),
$$

it follows that

$$
\tau_{2}(\phi)=(n-2)\left(\operatorname{Tr}_{g}\left(\nabla^{\phi}\right)^{2} d \phi(\operatorname{grad} \ln \lambda)+\operatorname{Tr}_{g} R^{N}(d \phi(\operatorname{grad} \ln \lambda), d \phi) d \phi\right) .
$$

By Lemma 2, we have

$$
\begin{aligned}
\operatorname{Tr}_{g}\left(\nabla^{\phi}\right)^{2} d \phi(\operatorname{grad} \ln \lambda) & =d \phi(\operatorname{grad} \Delta \ln \lambda)+2 d \phi\left(\operatorname{grad}\left(|\operatorname{grad} \ln \lambda|^{2}\right)\right) \\
& -(\Delta \ln \lambda) d \phi(\operatorname{grad} \ln \lambda)+d \phi\left(\operatorname{Ricci}^{M}(\operatorname{grad} \ln \lambda)\right) \\
& -(n-2)|\operatorname{grad} \ln \lambda|^{2} d \phi(\operatorname{grad} \ln \lambda) .
\end{aligned}
$$


By using lemma 3 and the fact that $\nabla_{\operatorname{grad}} \ln \lambda \operatorname{grad} \ln \lambda=\frac{1}{2} \operatorname{grad}\left(|\operatorname{grad} \ln \lambda|^{2}\right)$

$$
\begin{aligned}
\operatorname{Tr}_{g} R^{N}(d \phi(\operatorname{grad} \ln \lambda), d \phi) d \phi & =d \phi\left(\operatorname{Ricci}^{M}(\operatorname{grad} \ln \lambda)\right)-(\Delta \ln \lambda) d \phi(\operatorname{grad} \ln \lambda) \\
& -\frac{(n-2)}{2} d \phi\left(\operatorname{grad}\left(|\operatorname{grad} \ln \lambda|^{2}\right)\right) .
\end{aligned}
$$

If we replace (32) and (33) in (31), we deduce that

$$
\begin{aligned}
\tau_{2}(\phi) & =(n-2) d \phi(\operatorname{grad} \Delta \ln \lambda)-\frac{(n-2)(n-6)}{2} d \phi\left(\operatorname{grad}\left(|\operatorname{grad} \ln \lambda|^{2}\right)\right) \\
& -(n-2)\left(2(\Delta \ln \lambda)+(n-2)|\operatorname{grad} \ln \lambda|^{2}\right) d \phi(\operatorname{grad} \ln \lambda) \\
& +2(n-2) d \phi\left(\operatorname{Ricci}^{M}(\operatorname{grad} \ln \lambda)\right) .
\end{aligned}
$$

Then the bi-tension field of $\phi$ is given by :

$$
\tau_{2}(\phi)=(n-2) d \phi(H)
$$

where

$$
\begin{aligned}
H & =\operatorname{grad} \Delta \ln \lambda-\frac{(n-6)}{2} \operatorname{grad}\left(|\operatorname{grad} \ln \lambda|^{2}\right)+2 \operatorname{Ricci}^{M}(\operatorname{grad} \ln \lambda) \\
& -\left(2(\Delta \ln \lambda)+(n-2)|\operatorname{grad} \ln \lambda|^{2}\right) \operatorname{grad} \ln \lambda .
\end{aligned}
$$

The proof of Theorem 2 is complete. By application of Theorem 2, we get the following result (see [15]).

Theorem 3. ([12]) Let $\phi:\left(M^{n}, g\right) \rightarrow\left(N^{n}, h\right)(n \geq 3)$ to be a conformal map of dilation $\lambda$, then $\phi$ is biharmonic if and only if the dilation $\lambda$ satisfies

$$
\begin{aligned}
& \operatorname{grad}(\Delta \ln \lambda)-\left(2(\Delta \ln \lambda)+(n-2)|\operatorname{grad} \ln \lambda|^{2}\right) \operatorname{grad} \ln \lambda \\
& +\frac{6-n}{2} \operatorname{grad}\left(|\operatorname{grad} \ln \lambda|^{2}\right)+2 \operatorname{Ricci}^{M}(\operatorname{grad} \ln \lambda)=0 .
\end{aligned}
$$

In particular, we prove that the biharmonicity of the conformal map $\phi:\left(\mathbb{R}^{n}, g\right) \rightarrow\left(N^{n}, h\right)(n \geq 3)$ where the dilation $\lambda$ is radial $\left(\ln \lambda=\alpha(r), r=|x|\right.$ and $\left.\alpha \in C^{\infty}(\mathbb{R}, \mathbb{R})\right)$ is equivalent to an ordinary differential equation of the second order. More precisely, we have

Corollary 2. Let $\phi:\left(\mathbb{R}^{n}, g\right) \rightarrow\left(N^{n}, h\right)(n \geq 3)$ to be a conformal map of dilation $\lambda$ when we suppose that $\ln \lambda$ is radial $\left(\ln \lambda=\alpha(r), r=|x|\right.$ and $\left.\alpha \in C^{\infty}(\mathbb{R}, \mathbb{R})\right)$. Then $\phi$ is biharmonic if and only if $\beta=\alpha^{\prime}$ satisfies the following ordinary differential equation:

$$
\beta^{\prime \prime}-(n-4) \beta \beta^{\prime}+\frac{n-1}{r} \beta^{\prime}-\frac{n-1}{r^{2}} \beta-\frac{2(n-1)}{r} \beta^{2}-(n-2) \beta^{3}=0 .
$$

Proof of Corollary 2 Let $\phi:\left(\mathbb{R}^{n}, g\right) \rightarrow\left(N^{n}, h\right)(n \geq 3)$ to be a conformal map of dilation $\lambda$ such that $\ln \lambda=\alpha(r)$. By Theorem 3, $\phi$ is biharmonic if and only if the dilation $\lambda$ satisfies

$$
\begin{aligned}
& \operatorname{grad}(\Delta \ln \lambda)-\left(2(\Delta \ln \lambda)+(n-2)|\operatorname{grad} \ln \lambda|^{2}\right) \operatorname{grad} \ln \lambda \\
& +\frac{6-n}{2} \operatorname{grad}\left(|\operatorname{grad} \ln \lambda|^{2}\right)+2 \operatorname{Ricci}^{M}(\operatorname{grad} \ln \lambda)=0 .
\end{aligned}
$$

A direct calculation gives

$$
\operatorname{grad} \ln \lambda=\alpha^{\prime} \frac{\partial}{\partial r^{\prime}}
$$




$$
\begin{gathered}
|\operatorname{grad} \ln \lambda|^{2}=\left(\alpha^{\prime}\right)^{2} \\
\operatorname{grad}\left(|\operatorname{grad} \ln \lambda|^{2}\right)=2 \alpha^{\prime} \alpha^{\prime \prime} \frac{\partial}{\partial r^{\prime}} \\
\Delta \ln \lambda=\alpha^{\prime \prime}+\frac{n-1}{r} \alpha^{\prime}
\end{gathered}
$$

and

$$
\operatorname{grad}(\Delta \ln \lambda)=\left(\alpha^{\prime \prime \prime}+\frac{n-1}{r} \alpha^{\prime \prime}-\frac{n-1}{r^{2}} \alpha^{\prime}\right) \frac{\partial}{\partial r} .
$$

Therefore $\phi$ is biharmonic if and only if the function $\alpha$ satisfies the following differential equation

$$
\alpha^{\prime \prime \prime}-(n-4) \alpha^{\prime} \alpha^{\prime \prime}+\frac{n-1}{r} \alpha^{\prime \prime}-\frac{n-1}{r^{2}} \alpha^{\prime}-\frac{2(n-1)}{r}\left(\alpha^{\prime}\right)^{2}-(n-2)\left(\alpha^{\prime}\right)^{3}=0 .
$$

If we denote $\beta=\alpha^{\prime}$, the biharmonicity of $\phi$ is equivalent to the differential equation

$$
\beta^{\prime \prime}-(n-4) \beta \beta^{\prime}+\frac{n-1}{r} \beta^{\prime}-\frac{n-1}{r^{2}} \beta-\frac{2(n-1)}{r} \beta^{2}-(n-2) \beta^{3}=0 .
$$

As a consequence of the Corollary 2, We will present some remarks which we give a particular solutions of the equation (34) that allows us to construct a biharmonic non-harmonic maps.

Remark 3. . Looking for particular solutions of type $\beta=\frac{a}{r}\left(a \in \mathbb{R}^{*}\right)$. By (34), we deduce that $\phi:\left(\mathbb{R}^{n}, g\right) \rightarrow$ $\left(N^{n}, h\right)(n \geq 3)$ is biharmonic if and only if $a$ is a solution of the algebraic equation

$$
(n-2) a^{2}+(n+2) a+2 n-2=0 .
$$

This equation has real solutions if and only if $n \in\{3,4\}$.

1. If $n=3$, we find $a=\frac{-5+\sqrt{17}}{2}$ or $a=\frac{-5-\sqrt{17}}{2}$, so $\lambda=\mathrm{Cr}^{-\left(\frac{5-\sqrt{17}}{2}\right)}$ or $\lambda=\mathrm{Cr}^{-\left(\frac{5+\sqrt{17}}{2}\right)}\left(\mathrm{C} \in \mathbb{R}_{+}^{*}\right)$. It follows that any conformal map $\phi:\left(\mathbb{R}^{3}, g\right) \rightarrow\left(N^{3}, h\right)$ of dilation $\lambda=\mathrm{Cr}^{-\left(\frac{5-\sqrt{17}}{2}\right)}$ or $\lambda=\mathrm{Cr}^{-\left(\frac{5+\sqrt{17}}{2}\right)}$ is biharmonic non-harmonic.

2. If $n=4$, we find $a=-1$ or $a=-2$, so $\lambda=\frac{C}{r^{2}}$ or $\lambda=\frac{C}{r}\left(C \in \mathbb{R}_{+}^{*}\right)$. Then, in this case any conformal map $\phi:\left(\mathbb{R}^{4}, g\right) \rightarrow\left(N^{4}, h\right)$ of dilation $\lambda=\frac{C}{r^{2}}$ or $\lambda=\frac{C}{r}$ is biharmonic non-harmonic. For example, the inversion $\phi:\left(\mathbb{R}^{n} \backslash\{0\}, g_{\mathbb{R}^{n}}\right) \longrightarrow\left(\mathbb{R}^{n} \backslash\{0\}, g_{\mathbb{R}^{n}}\right)$ definded by $\phi(x)=\frac{x}{|x|^{2}}$ is a conformal map with dilation $\lambda=\frac{1}{r^{2}}$. By (34), the inversion is biharmonic non-harmonic if and only if $n=4$.

Remark 4. . Looking for particular solutions of type $\beta=\frac{a r}{1+r^{2}}\left(a \in \mathbb{R}^{*}\right)$. By (34), $\phi:\left(\mathbb{R}^{n}, g\right) \rightarrow\left(N^{n}, h\right)$ $(n \geq 3)$ is biharmonic if and only we have

$$
(n-2) a^{2}+(n+2) a+2 n-2=0
$$

and

$$
3(n-2) a+2 n+4=0 .
$$

These two equations gives $a=-2$ and $n=4$, it follows that the dilation is equal to $\lambda=\frac{C}{r^{2}+1}\left(C \in \mathbb{R}_{+}^{*}\right)$. Then, all conformal maps $\phi:\left(\mathbb{R}^{4}, g\right) \rightarrow\left(N^{4}, h\right)$ of dilation $\lambda=\frac{C}{r^{2}+1}$ are biharmonic non-harmonic. For example, the inverse of the stereographic projection of the sphere $\phi: \mathbb{R}^{n} \longrightarrow S^{n}$ definded by $\phi(x)=\frac{1}{|x|^{2}+1}\left(|x|^{2}-1,2 x\right)$ is a conformal map with dilation $\lambda=\frac{2}{r^{2}+1}$. By (34), the inverse of the stereographic projection is biharmonic non-harmonic if and only if $n=4$. 
The last part of this paper is devoted to the study of biharmonic maps between warped product manifolds, these maps were also studied in [17]. We will give some results of the biharmonicity in other particular cases.

\section{Biharmonic maps and the warped product}

Let $\left(M^{m}, g\right)$ and $\left(N^{n}, h\right)$ two Riemannian manifolds and let $f \in C^{\infty}(M)$ be a positive function. The warped product $M \times_{f} N$ is the product manifolds $M \times N$ endowed with the Riemannian metric $G_{f}$ defined, for $X, Y \in \Gamma(T(M \times N))$, by

$$
G_{f}(X, Y)=g(d \pi(X), d \pi(Y))+(f \circ \pi)^{2} h(d \eta(X), d \eta(Y)),
$$

where $\pi: M \times N \longrightarrow M$ and $\eta: M \times N \longrightarrow N$ are respectively the first and the second projection. The function $f$ is called the warping function of the warped product. Let $X, Y \in \Gamma(T(M \times N))$, $X=\left(X_{1}, X_{2}\right), Y=\left(Y_{1}, Y_{2}\right)$. Denote by $\nabla$ the Levi-Civita connection on the Riemannian product $M \times N$. The Levi-Civita connection $\widetilde{\nabla}$ of the warped product $M \times{ }_{f} N$ is given by

$$
\widetilde{\nabla}_{X} Y=\nabla_{X} Y+X_{1}(\ln f)\left(0, Y_{2}\right)+Y_{1}(\ln f)\left(0, X_{2}\right)-f^{2} h\left(X_{2}, Y_{2}\right)(\operatorname{grad} \ln f, 0) .
$$

In the first, we consider a smooth map $\phi:\left(M^{m}, g\right) \longrightarrow\left(P^{p}, k\right)$ and we defined the map $\widetilde{\phi}$ : $\left(M^{m} \times_{f} N^{n}, G_{f}\right) \longrightarrow\left(P^{p}, k\right)$ by $\widetilde{\phi}(x, y)=\phi(x)$. We will study the biharmonicity of $\widetilde{\phi}$. By calculating the tension field of $\widetilde{\phi}$, we obtain the following result :

Proposition 2. Let $\phi:\left(M^{m}, g\right) \longrightarrow\left(P^{p}, k\right)$ be a smooth map. The tension field of the map $\widetilde{\phi}$ : $\left(M^{m} \times{ }_{f} N^{n}, G_{f}\right) \longrightarrow\left(P^{p}, k\right)$ defined by $\widetilde{\phi}(x, y)=\phi(x)$ is given by

$$
\tau(\widetilde{\phi})=\tau(\phi)+n d \phi(\operatorname{grad} \ln f)
$$

Proof of Proposition 2. Let us choose $\left\{e_{i}\right\}_{1 \leq i \leq m}$ to be an orthonormal frame on $M$ and $\left\{f_{j}\right\}_{1 \leq j \leq n}$ to be an orthonormal frame on $N$. An orthonormal frame on $M \times_{f} N$ is given by $\left\{\left(e_{i}, 0\right), \frac{1}{f}\left(0, f_{j}\right)\right\}$. Note that in this case we have $d \widetilde{\phi}(X, Y)=(d \phi(X), 0)$ for any $X \in \Gamma(T M)$ and $Y \in \Gamma(T N)$. By definition to the tension field, we have

$$
\begin{aligned}
\tau(\widetilde{\phi}) & =\operatorname{Tr}_{G_{f}} \nabla d \widetilde{\phi} \\
& =\nabla_{\left(e_{i}, 0\right)}^{\widetilde{\phi}} d \widetilde{\phi}\left(e_{i}, 0\right)+\frac{1}{f^{2}} \nabla_{\left(0, f_{j}\right)}^{\widetilde{\phi}} d \widetilde{\phi}\left(0, f_{j}\right) \\
& -d \widetilde{\phi}\left(\widetilde{\nabla}_{\left(e_{i}, 0\right)}\left(e_{i}, 0\right)\right)-\frac{1}{f^{2}} d \widetilde{\phi}\left(\widetilde{\nabla}_{\left(0, f_{j}\right)}\left(0, f_{j}\right)\right) .
\end{aligned}
$$

A simple calculation gives

$$
\nabla_{\left(e_{i}, 0\right)}^{\widetilde{\Phi}} d \widetilde{\phi}\left(e_{i}, 0\right)=\nabla_{e_{i}}^{\phi} d \phi\left(e_{i}\right)
$$

and

$$
\nabla_{\left(0, f_{j}\right)}^{\widetilde{\Phi}} d \widetilde{\phi}\left(0, f_{j}\right)=0,
$$

By using the equation (35), we deduce that

$$
\widetilde{\nabla}_{\left(e_{i}, 0\right)}\left(e_{i}, 0\right)=\left(\nabla_{e_{i}} e_{i}, 0\right)
$$

and

$$
\widetilde{\nabla}_{\left(0, f_{j}\right)}\left(0, f_{j}\right)=\left(0, \nabla_{f_{j}} f_{j}\right)-n f^{2}(\operatorname{grad} \ln f, 0) .
$$


It follows that

$$
\tau(\widetilde{\phi})=\nabla_{e_{i}}^{\phi} d \phi\left(e_{i}\right)-d \phi\left(\nabla_{e_{i}}^{M} e_{i}\right)+n d \phi(\operatorname{grad} \ln f)
$$

then, we obtain

$$
\tau(\widetilde{\phi})=\tau(\phi)+n d \phi(\operatorname{grad} \ln f) .
$$

Remark 5. If $\phi:\left(M^{m}, g\right) \longrightarrow\left(P^{m}, k\right)(m \geq 3)$ is a conformal map with dilation $\lambda$, the tension field of $\widetilde{\phi}$ is given by

$$
\tau(\widetilde{\phi})=(2-m) d \phi(\operatorname{grad} \ln \lambda)+n d \phi(\operatorname{grad} \ln f)=d \phi\left(\operatorname{grad} \ln \left(\lambda^{2-m} f^{n}\right)\right) .
$$

Then $\widetilde{\phi}$ is harmonic if and only if the function $\lambda^{2-m} f^{n}$ is constant.

We will now calculate the bitension field of the map $\widetilde{\phi}:\left(M^{m} \times_{f} N^{n}, G_{f}\right) \longrightarrow\left(P^{p}, k\right)$.

Theorem 4. Let $\phi:\left(M^{m}, g\right) \longrightarrow\left(P^{p}, k\right)$ be a smooth map. The bitension field of the map $\widetilde{\phi}$ : $\left(M^{m} \times_{f} N^{n}, G_{f}\right) \longrightarrow\left(P^{p}, k\right)$ defined by $\widetilde{\phi}(x, y)=\phi(x)$ is given by

$$
\begin{aligned}
\tau_{2}(\widetilde{\phi}) & =\tau_{2}(\phi)-n\left(\operatorname{Tr}_{g} \nabla^{2} d \phi(\operatorname{grad} \ln f)+\operatorname{Tr}_{g} R^{p}(d \phi(\operatorname{grad} \ln f), d \phi) d \phi\right) \\
& -n \nabla_{g \operatorname{grad} \ln f} \tau(\phi)-n^{2} \nabla_{\operatorname{grad} \ln f} d \phi(\operatorname{grad} \ln f) .
\end{aligned}
$$

Proof of Theorem 4. By definition of the bi-tension field, we have

$$
\tau_{2}(\widetilde{\phi})=-\operatorname{Tr}_{G_{f}}\left(\nabla^{\widetilde{\phi}}\right)^{2} \tau(\widetilde{\phi})-\operatorname{Tr}_{G_{f}} R^{P}(\tau(\widetilde{\phi}), d \widetilde{\phi}) d \widetilde{\phi}
$$

For the first term $\operatorname{Tr}_{G_{f}}\left(\nabla^{\widetilde{\phi}}\right)^{2} \tau(\widetilde{\phi})$, we have

$$
\begin{aligned}
& \operatorname{Tr}_{G_{f}}\left(\nabla^{\widetilde{\phi}}\right)^{2} \tau(\widetilde{\phi})=\nabla_{\left(e_{i}, 0\right)}^{\widetilde{\phi}} \nabla_{\left(e_{i}, 0\right)}^{\widetilde{\phi}} \tau(\widetilde{\phi})+\frac{1}{f^{2}} \nabla_{\left(0, f_{j}\right)}^{\widetilde{\phi}} \nabla_{\left(0, f_{j}\right)}^{\widetilde{\phi}} \tau(\widetilde{\phi})
\end{aligned}
$$

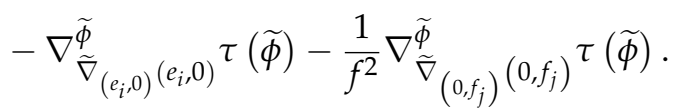

We will study term by term the right-hand of this expression. A simple calculation gives

$$
\begin{aligned}
\nabla_{\left(e_{i}, 0\right)}^{\widetilde{\phi}} \nabla_{\left(e_{i}, 0\right)}^{\widetilde{\phi}} \tau(\widetilde{\phi}) & =\nabla_{\left(e_{i}, 0\right)}^{\widetilde{\phi}} \nabla_{\left(e_{i}, 0\right)}^{\widetilde{\phi}} \tau(\phi)+n \nabla_{\left(e_{i}, 0\right)}^{\widetilde{\phi}} \nabla_{\left(e_{i}, 0\right)}^{\widetilde{\phi}} d \phi(\operatorname{grad} \ln f) \\
& =\nabla_{e_{i}}^{\phi} \nabla_{e_{i}}^{\phi} \tau(\phi)+n \nabla_{e_{i}}^{\phi} \nabla_{e_{i}}^{\phi} d \phi(\operatorname{grad} \ln f)
\end{aligned}
$$

and

$$
\nabla_{\left(0, f_{j}\right)}^{\widetilde{\phi}} \nabla_{\left(0, f_{j}\right)}^{\widetilde{\phi}} \tau(\widetilde{\phi})=0
$$

By using the equation (35), we obtain

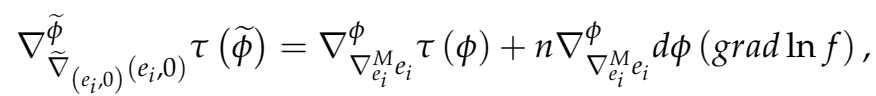

and

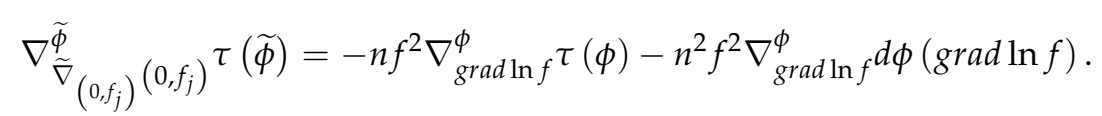

Then, we deduce that

$$
\begin{aligned}
\operatorname{Tr}_{G_{f}}\left(\nabla^{\widetilde{\phi}}\right)^{2} \tau(\widetilde{\phi}) & =\operatorname{Tr}_{g}\left(\nabla^{\phi}\right)^{2} \tau(\phi)+n \operatorname{Tr}_{g}\left(\nabla^{\phi}\right)^{2} d \phi(\operatorname{grad} \ln f) \\
& +n \nabla_{g \operatorname{grad} \ln f}^{\phi} \tau(\phi)+n^{2} \nabla_{\operatorname{grad} \ln f}^{\phi} d \phi(\operatorname{grad} \ln f)
\end{aligned}
$$


To complete the proof, we will simplify the term $\operatorname{Tr}_{G_{f}} R^{P}(\tau(\widetilde{\phi}), d \widetilde{\phi}) d \widetilde{\phi}$, we have

$$
\begin{aligned}
\operatorname{Tr}_{G_{f}} R^{P}(\tau(\widetilde{\phi}), d \widetilde{\phi}) d \widetilde{\phi} & =R^{P}\left(\tau(\widetilde{\phi}), d \widetilde{\phi}\left(e_{i}, 0\right)\right) d \widetilde{\phi}\left(e_{i}, 0\right) \\
& +\frac{1}{f^{2}} R^{P}\left(\tau(\widetilde{\phi}), d \widetilde{\phi}\left(0, f_{j}\right)\right) d \widetilde{\phi}\left(0, f_{j}\right) \\
& =R^{P}\left(\tau(\widetilde{\phi}), d \widetilde{\phi}\left(e_{i}, 0\right)\right) d \widetilde{\phi}\left(e_{i}, 0\right) \\
& =R^{P}\left(\tau(\phi), d \phi\left(e_{i}\right)\right) d \phi\left(e_{i}\right) \\
& +n R^{P}\left(d \phi(\operatorname{grad} \ln f), d \phi\left(e_{i}\right)\right) d \phi\left(e_{i}\right) .
\end{aligned}
$$

It follows that

$$
\operatorname{Tr}_{G_{f}} R^{P}(\tau(\widetilde{\phi}), d \widetilde{\phi}) d \widetilde{\phi}=\operatorname{Tr}_{g} R^{P}(\tau(\phi), d \phi) d \phi+n \operatorname{Tr}_{g} R^{P}(d \phi(\operatorname{grad} \ln f), d \phi) d \phi .
$$

If we replace (39) and (40) in (38), we obtain

$$
\begin{aligned}
\tau_{2}(\widetilde{\phi}) & =\tau_{2}(\phi)-n\left(\operatorname{Tr}_{g} \nabla^{2} d \phi(\operatorname{grad} \ln f)+\operatorname{Tr}_{g} R^{p}(d \phi(\operatorname{grad} \ln f), d \phi) d \phi\right) \\
& -n \nabla_{\operatorname{grad} \ln f} \tau(\phi)-n^{2} \nabla_{\operatorname{grad} \ln f} d \phi(\operatorname{grad} \ln f) .
\end{aligned}
$$

The proof of Theorem 4 is complete.

Remark 6. Theorem 4 is a particular result of generalized warped product manifolds (see [18]).

As a consequence, if $\phi$ is harmonic, we have

Corollary 3. Let $\phi:\left(M^{m}, g\right) \longrightarrow\left(P^{p}, k\right)$ a harmonic map. The map $\widetilde{\phi}:\left(M^{m} \times_{f} N^{n}, G_{f}\right) \longrightarrow\left(P^{p}, k\right)$ defined by $\widetilde{\phi}(x, y)=\phi(x)$ is biharmonic if and only if

$$
\operatorname{Tr}_{g} \nabla^{2} d \phi(\operatorname{grad} \ln f)+\operatorname{Tr}_{g} R^{P}(d \phi(\operatorname{grad} \ln f), d \phi) d \phi+n \nabla_{\operatorname{grad} \ln f} d \phi(\operatorname{grad} \ln f)=0 .
$$

In particular if $\phi=I d_{M}$, the first projection $P_{1}:\left(M^{m} \times_{f} N^{n}, G_{f}\right) \longrightarrow\left(M^{m}, g\right)$ defined by $P_{1}(x, y)=x$ is biharmonic if and only if (see [17])

$$
\operatorname{grad} \Delta \ln f+\frac{n}{2} \operatorname{grad}\left(|\operatorname{grad} \ln f|^{2}\right)+2 \operatorname{Ricci}^{M}(\operatorname{grad} \ln f)=0 .
$$

In the following we shall present an example of biharmonic non-harmonic maps.

Example 1. Let $\widetilde{\varphi}: \mathbb{R}^{m} \backslash\{0\} \times_{f} N^{n} \longrightarrow \mathbb{R}^{m} \backslash\{0\}$ defined by $\widetilde{\varphi}(x, y)=\frac{x}{|x|^{2}}$ when we suppose that $\ln f$ is radial $(\ln f=\alpha(r))$. Then by Theorem 4, we deduce that the map $\widetilde{\varphi}: \mathbb{R}^{m} \backslash\{0\} \times{ }_{f} N^{n} \longrightarrow \mathbb{R}^{m} \backslash\{0\}$ is biharmonic if and only if the function $\alpha$ satisfies the following differential equation

$$
n \alpha^{\prime \prime \prime}+\frac{n(m-5)}{r} \alpha^{\prime \prime}-\frac{3 n(3 m-7)}{r^{2}} \alpha^{\prime}+n^{2} \alpha^{\prime} \alpha^{\prime \prime}-\frac{2 n^{2}}{r}\left(\alpha^{\prime}\right)^{2}-\frac{8(m-2)(m-4)}{r^{3}}=0 .
$$

Let $\beta=\alpha^{\prime}$, this equation becomes

$$
n \beta^{\prime \prime}+\frac{n(m-5)}{r} \beta^{\prime}-\frac{3 n(3 m-7)}{r^{2}} \beta+n^{2} \beta \beta^{\prime}-\frac{2 n^{2}}{r} \beta^{2}-\frac{8(m-2)(m-4)}{r^{3}}=0 .
$$

Looking for particular solutions of type $\beta=\frac{a}{r}\left(a \in \mathbb{R}^{*}\right)$, then $\widetilde{\varphi}: \mathbb{R}^{m} \backslash\{0\} \times_{f} N^{n} \longrightarrow \mathbb{R}^{m} \backslash\{0\}$ is biharmonic if and only if

$$
3 n^{2} a^{2}+2 n(5 m-14) a+8(m-2)(m-4)=0 .
$$

This equation has two solutions $a=\frac{4-2 m}{n}$ and $a=\frac{4(4-m)}{3 n}$. 
1. For $a=\frac{4-2 m}{n}$, we obtain $f(r)=C r^{\frac{4-2 m}{n}}$ and in this case $\widetilde{\varphi}: \mathbb{R}^{m} \backslash\{0\} \times_{f} N^{n} \longrightarrow \mathbb{R}^{m} \backslash\{0\}$ is harmonic so biharmonic.

2. For $a=\frac{4(4-m)}{3 n}$, we obtain $f(r)=\operatorname{Cr} \frac{4(4-m)}{3 n}$ and in this case $\widetilde{\varphi}: \mathbb{R}^{m} \backslash\{0\} \times_{f} N^{n} \longrightarrow \mathbb{R}^{m} \backslash\{0\}$ is biharmonic non-harmonic.

Now, we consider a smooth map $\psi:\left(N^{n}, g\right) \longrightarrow\left(P^{p}, k\right)$ and we define the map $\widetilde{\psi}$ : $\left(M^{m} \times_{f} N^{n}, G_{f}\right) \longrightarrow\left(P^{p}, k\right)$ by $\widetilde{\psi}(x, y)=\psi(y)$. We will study the biharmonicity of $\widetilde{\psi}$, we obtain the following result :

Theorem 5. Let $\psi:\left(N^{n}, h\right) \rightarrow\left(P^{p}, k\right)$ be a smooth map, we define $\widetilde{\psi}:\left(M^{m} \times{ }_{f^{2}} N^{n}, G_{f^{2}}\right) \rightarrow\left(P^{p}, k\right)$ by $\widetilde{\psi}(x, y)=\psi(y)$. The tension field and the bi-tension field of $\widetilde{\psi}$ are given by

$$
\tau(\widetilde{\psi})=\frac{1}{f^{2} \circ \pi} \tau(\psi)
$$

and

$$
\tau_{2}(\widetilde{\psi})=\frac{1}{f^{4} \circ \pi} \tau_{2}(\psi)-\frac{2}{f^{2} \circ \pi}\left(\left(\Delta \ln f+(n-2)|\operatorname{grad} \ln f|^{2}\right) \circ \pi\right) \tau(\psi) .
$$

Proof of Theorem 5. In the first, we calculate the tension field of of $\widetilde{\psi}$. By definition to the tension field, we have

$$
\begin{aligned}
\tau(\widetilde{\psi}) & =\operatorname{Tr}_{G_{f}} \nabla d \widetilde{\psi} \\
& =\nabla_{\left(e_{i}, 0\right)}^{\widetilde{\psi}} d \widetilde{\psi}\left(e_{i}, 0\right)+\frac{1}{f^{2} \circ \pi} \nabla_{\left(0, f_{j}\right)}^{\widetilde{\psi}} d \widetilde{\psi}\left(0, f_{j}\right) \\
& -d \widetilde{\psi}\left(\widetilde{\nabla}_{\left(e_{i}, 0\right)}\left(e_{i}, 0\right)\right)-\frac{1}{f^{2} \circ \pi} d \widetilde{\psi}\left(\widetilde{\nabla}_{\left(0, f_{j}\right)}\left(0, f_{j}\right)\right) .
\end{aligned}
$$

By using the equation (35), we obtain

$$
\tau(\widetilde{\psi})=\frac{1}{f^{2} \circ \pi} \nabla_{f_{j}}^{\psi} d \psi\left(f_{j}\right)-\frac{1}{f^{2} \circ \pi} d \psi\left(\nabla_{f_{j}} f_{j}\right)=\frac{1}{f^{2} \circ \pi} \tau(\psi),
$$

then

$$
\tau(\widetilde{\psi})=\frac{1}{f^{2} \circ \pi} \tau(\psi) .
$$

By this expression, we deduce that $\widetilde{\psi}$ is harmonic if and only if $\psi$ is harmonic. Now, we will calculate the bi-tension field of $\widetilde{\psi}$. By definition, we have

$$
\tau_{2}(\widetilde{\psi})=-\operatorname{Tr}_{G_{f}}\left(\nabla^{\widetilde{\psi}}\right)^{2} \tau(\widetilde{\psi})-\operatorname{Tr}_{G_{f}} R^{P}(\tau(\widetilde{\psi}), d \widetilde{\psi}) d \widetilde{\psi}
$$

For the first term $\operatorname{Tr}_{G_{f}}\left(\nabla^{\widetilde{\psi}}\right)^{2} \tau(\widetilde{\psi})$, we have

$$
\begin{aligned}
& \operatorname{Tr}_{G_{f}}\left(\nabla^{\widetilde{\psi}}\right)^{2} \tau(\widetilde{\psi})=\nabla_{\left(e_{i}, 0\right)}^{\widetilde{\psi}} \nabla_{\left(e_{i}, 0\right)}^{\widetilde{\psi}} \tau(\widetilde{\psi})+\frac{1}{f^{2} \circ \pi} \nabla_{\left(0, f_{j}\right)}^{\widetilde{\psi}} \nabla_{\left(0, f_{j}\right)}^{\widetilde{\psi}} \tau(\widetilde{\psi}) \\
&-\nabla_{\widetilde{\nabla}_{\left(e_{i}, 0\right)}\left(e_{i}, 0\right)}^{\widetilde{\psi}} \tau(\widetilde{\psi})-\frac{1}{f^{2} \circ \pi} \nabla_{\widetilde{\nabla}}^{\widetilde{\psi}}{ }_{\left(0, f_{j}\right)}\left(0, f_{j}\right) \\
& \tau(\widetilde{\psi})
\end{aligned}
$$

A long calculation gives

$$
\nabla_{\left(e_{i}, 0\right)}^{\widetilde{\widetilde{T}}} \nabla_{\left(e_{i}, 0\right)}^{\widetilde{\psi}} \tau(\widetilde{\psi})=\frac{2}{f^{2} \circ \pi}\left(\left(2|\operatorname{grad} \ln f|^{2}-e_{i}\left(e_{i}(\ln f)\right)\right) \circ \pi\right) \tau(\psi)
$$

and

$$
\frac{1}{f^{2} \circ \pi} \nabla_{\left(0, f_{j}\right)}^{\widetilde{\psi}} \nabla_{\left(0, f_{j}\right)}^{\widetilde{\psi}} \tau(\widetilde{\psi})=\frac{1}{f^{4} \circ \pi} \nabla_{f_{j}}^{\psi} \nabla_{f_{j}}^{\psi} \tau(\psi)
$$


Finally, by (35), we obtain

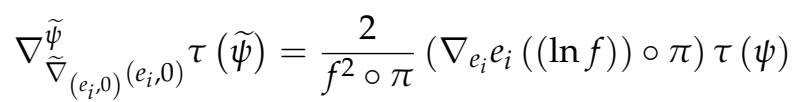

and

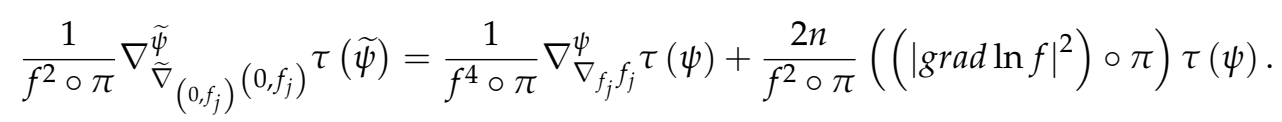

Which gives us

$$
\operatorname{Tr}_{G_{f}}(\nabla \widetilde{\psi})^{2} \tau(\widetilde{\psi})=\frac{1}{f^{4} \circ \pi} \operatorname{Tr}_{h} \nabla^{2} \tau(\psi)-\frac{2}{f^{2} \circ \pi}\left(\left(\Delta \ln f+(n-2)|\operatorname{grad} \ln f|^{2}\right) \circ \pi\right) \tau(\psi)
$$

Finally for the first term $\operatorname{Tr}_{G_{f}} R^{P}(\tau(\widetilde{\psi}), d \widetilde{\psi}) d \widetilde{\psi}$, it is easy to verify that

$$
\operatorname{Tr}_{G_{f}} R^{P}(\tau(\widetilde{\psi}), d \widetilde{\psi}) d \widetilde{\psi}=\frac{1}{f^{4} \circ \pi} \operatorname{Tr}_{h} R^{P}(\tau(\psi), d \psi) d \psi
$$

If we substitute (44) and (45) in (43), we obtain

$$
\tau_{2}(\widetilde{\psi})=\frac{1}{f^{4} \circ \pi} \tau_{2}(\psi)-\frac{2}{f^{2} \circ \pi}\left(\left(\Delta \ln f+(n-2)|\operatorname{grad} \ln f|^{2}\right) \circ \pi\right) \tau(\psi) .
$$

This completes the proof of Theorem 5. An immediate consequence of Theorem 5 is given by the following corollary :

Corollary 4. Let $\psi:\left(N^{n}, h\right) \longrightarrow\left(P^{p}, k\right)$ a biharmonic non-harmonic map. The map $\widetilde{\phi}$ : $\left(M^{m} \times_{f} N^{n}, G_{f^{2}}\right) \longrightarrow\left(P^{p}, k\right)$ defined by $\widetilde{\psi}(x, y)=\psi(y)$ is biharmonic if and only if the function $f^{n-2}$ is harmonic.

Acknowledgments: The authors would like to thank the referee for some useful comments and their helpful suggestions that have improved the quality of this paper.

Author Contributions: The authors provide equal contributions to this paper.

Conflicts of Interest: The authors declare no conflict of interest.

\section{References}

1. Eells, J.; Lemaire, L. A report on harmonic maps. Bull. Lond. Math. Soc. 1978, 16, 1-68.

2. Eells, J.; Lemaire, L. Another report on harmonic maps. Bull. Lond. Math. Soc. 1988, 20, 385-524.

3. Eells, J.; Lemaire, L. Selected topics in harmonic maps; CBMS Regional Conference Series in Mathematics; American Mathematical Society: Providence, RI, USA, 1981; Vol. 150.

4. Eells, J.; Ratto, A. Harmonic Maps and Minimal Immersions with Symmetries: Methods of Ordinary Differential Equations Applied to Elliptic Variational Problems; Princeton University Press: Princeton, NJ, USA, 1993; Vol. 130.

5. Baird, P.; Eells, J. A Conservation Law for Harmonic Maps; Lecture Notes in Math. 894; Springer : Berlin, Germany, 1981; pp. 1-25.

6. Jiang, G.Y. 2-harmonic maps and their first and second variational formulas. Chin. Ann. Math. Ser. 1986, A7, 389-402.

7. Loubeau, E.; Montaldo, S.; Oniciuc, C. The stress-energy tensor for biharmonic maps. Math. Z. 2008, 259, 503-524.

8. Baird, P.; Kamissoko, D. On constructing biharmonic maps and metrics. Ann. Glob. Anal. Geom. 2003, 23, 65-75.

9. Balmus, A. Biharmonic properties and conformal changes. Analele Stiintifice ale Univ. Al.I. Cuza Iasi Mat. 2004, 50, 367-372. 
10. Ou, Y.-L. p-harmonic morphisms, biharmonic morphisms, and non-harmonic biharmonic maps. J. Geom. Phys. 2006, 56, 358-374.

11. Ouakkas, S. Biharmonic maps, conformal deformations and the Hopf maps. Diff. Geom. Appl. 2008, 26, 495-502.

12. Baird, P.; Fardoun, A.; Ouakkas, S. Conformal and semi-conformal biharmonic maps. Ann. Glob. Anal. Geom. 2008, 34, 403-414.

13. Montaldo, S.; Oniciuc, C. A short survey of biharmonic maps between Riemannian manifolds. Rev. Union Mat. Argent. 2006, 47, 1-22.

14. Baird, P. Harmonic Maps with Symmetry, Harmonic Morphisms and Deformation of Metrics; Research Notes in Mathematics; CRC Press: London, UK,1983; pp. 27-39.

15. Baird, P.; Loubeau, E.; Oniciuc, C. Harmonic and biharmonic maps from surfaces. In Harmonic maps and differential geometry; Amer. Math. Soc.: Providence, RI, USA, 2011; pp. 234-241.

16. Baird, P.; Wood, J.C. Harmonic Morphisms between Riemannain Manifolds; London mathematical Society Monographs (N.S.); Oxford University Press: Oxford, UK, 2003.

17. Balmus, A.; Montaldo, S.; Oniciuc, C. Biharmonic maps between warped product manifolds. J. Geom. Phys. 2007, 57, 449-466.

18. Djaa, N.E.H.; Boulal, A.; Zagane, A. Generalized warped product manifolds and biharmonic maps. Acta Math. Univ. Comen. 2012, 81, 283-298.

(C) 2016 by the authors; licensee MDPI, Basel, Switzerland. This article is an open access article distributed under the terms and conditions of the Creative Commons by Attribution (CC-BY) license (http://creativecommons.org/licenses/by/4.0/). 